\title{
Separate determination of thickness and optical parameters by surface plasmon resonance: accuracy consideration
}

\author{
O.V. Rengevych, Yu.M. Shirshov, Yu.V. Ushenin, A.G. Beketov* \\ Institute of Semiconductor Physics of NASU, 45, prospect Nauki, 252028 Kiev, Ukraine. Tel.: (38044) 26518 27; \\ Fax: (38044) 26533 37; e-mail: rengevych@isp.kiev.ua \\ * Moscow Physico-Technical Institute, Pervomayskaya str. 30/1, 141700 Dolgoprudnyi, Moscow distr., Russia e-mail: beketov@mail.ru
}

\begin{abstract}
Reliability and precision of characterization of surface layers by SPR method was evaluated with relation to the experimental conditions and the strategy of extracting the film parameters. Consideration is bound up with sensor applications of SPR phenomenon and focused at problems of separate extraction of optical constants and thickness of the layer and determination of the total quantity of material constituting the surface coverage.

Computational scheme for modeling the SPR resonance for multilayer assembly, based on the Abeles matrix formalism, is presented. It is demonstrated that improper choice of the angle range the measurements are taken over may result in ambiguity in determination of the real part of the refractive index $n$ and the film thickness $d$. Nevertheless, the total quantity of material in the film can be estimated with reasonable accuracy even when correct separate extraction of $n$ and $d$ parameters is hampered by experimental errors and inadequacy of theoretical model of layered system.
\end{abstract}

Keywords: surface plasmon resonance, optical sensors, optical constants.

Paper received 28.12.98; revised manuscript received 09.07.99; accepted for publication 12.07.99.

\section{Introduction}

Surface plasmon resonance (SPR) spectroscopy enjoys rapidly growing applications in characterization of thin surface films, especially in fields of oligomolecular, biomolecular, and polymer studies and in biosensor technology. This technique is based on linear interaction between the evanescent electromagnetic wave of surface plasmons (SP) excited in thin metallic films and the surrounding media [1-3]. The major advantage of SPR over other all-optical techniques of comparable performances (ellipsometry, planar waveguide interferometry etc.) is a unique combination of an extremely high sensitivity to optical properties of surface layers and the ease of their realtime continuous monitoring. This feature makes SPR a useful tool for studying kinetics of interfacial chemical reactions and physical processes [4,5], especially when only qualitative data are required. However, in many instances such as adsorption and self-assembly of oligomolecules, surface polymerization studies etc. kinetics of the process generally depends on the surface layer growth mode and may differ drastically for monolayer, multilayer, or island growth. Therefore, possibility of separate evaluating of optical and geometrical parameters of surface layers is also of importance [5,6]. Unfortunately, these parameters can be extracted from the experimental data provided by SPR measurements in an indirect way only that requires a correct measurement procedure and subsequent mathematical processing of the results. Difficulties encountered when using this approach are similar to that when treating the ellipsometric data and are associated with two major problems [7]. First, an adequate theoretical consideration is required to describe propagation of electromagnetic waves in the surface layered structure to a high degree of approximation, while the only manageable model elaborated till now is restricted to an idealized system of isotropic homogeneous parallel layers. This means that effective values of 


\section{O.V. Rengevych et al.: Separate determination of thickness and...}

optical parameters and thickness only can be determined with this model.

Another problem is associated with limitations inherent in the SPR experiment as itself. Consider an attenuated total reflection (ATR) prism coupling in the Kretschmann - Raether geometry [8,9] for excitation of SPs and measurement of reflected intensity versus angle of incidence $\theta$. The surface plasmon resonance manifests itself as a dip in the angular reflectivity dependency at the resonance angle $\theta_{R}$, usually called the resonant curve. In addition to $\theta_{R}$, this curve is characterized by the halfwidth $\Delta \theta_{1 / 2}$, and the reflectivity value in the minimum $R_{\min }$. According to general considerations, these three parameters allow to find simultaneously up to three parameters of surface layers, provided that corresponding mathematical dependencies are non-degenerate. Therefore numerous studies in metallooptics were based on SPR technique [10-12]. In particular, it was used to determine optical parameters of $\mathrm{Au}$ thin films that are especially suited for SPR experiments [12]. But, as it will be demonstrated below, this approach may give ambiguous results even for idealized metal film fully characterized by complex refractive index $N=n$-ik and thickness $d$. Increasing complexity of surface layered system, for instance in the case of molecular adsorption, requires a part of model parameters to be determined independently. This requirement can in principle be accomplished by separate measuring of metal film parameters and then using their values for treatment of adsorption measurements. But, as can be shown by comparison of reference spectral dependencies of optical constants of gold [13-15] (Fig.1), there is significant controversy between different experimental data. It is evident that errors in metal film parameters will deteriorate the accuracy of characterization of adsorbed layers. Inaccuracy of metal parameters may originate from instrumental errors, divergence of real metal film properties from those predicted by the idealized model, inadequate treatment of free electron gas behavior when interacting with the electromagnetic wave, and, finally, improper choice of measurement conditions.

The purpose of this study is to evaluate both theoretically and experimentally the reliability of metal and dielec- tric film characterization by SPR method depending on experimental conditions and strategy of extracting the film parameters.

\section{Theory}

Capability of surface plasmons for probing the refractive index of the surrounding media within close vicinity to the metal surface is a consequence of a steep decay of the electromagnetic filed with distance from the interface. The penetration depth of the electromagnetic field into the surrounding medium depends on the normal component of its wavevector $k$ and, in the case of the Kretschmann - Raether geometry, decreases with increasing the angle of incidence onto the interface. Since the SPR resonant curve is of considerable angular width, typically from 3-5 to 10-15 degrees, penetration depth varies to some extent over this range. Therefore the resonant curve for the homogeneous surrounding medium will differ in shape from that for the layered structure. This effect is a prerequisite to determination of the overlayer thickness separately from its optical constants from the SPR measurements. This can best be done using fitting the experimental curve by the calculated one provided that an adequate model for the surface layers is available.

Consideration presented here is restricted to an idealized model of surface layered structure that consists of a semi-infinite half-space representing the prism, a set of parallel layers, and a half-space representing the surrounding medium (Fig. 2). All the media are supposed to be homogeneous and isotropic, and the interface boundaries to be flat and smooth. Optical properties of all the media constituting this structure are described by complex refractive indices. This approximation neglects contribution of nonlocal effects to the relationship between the electric field and the dielectric response of free electron gas in the metals, and, consequently, the spatial dispersion of optical constants of the metal layer $[10,11]$. Although the validity of these approximations is not evident, they are of common practice when treating the SPR measurements.

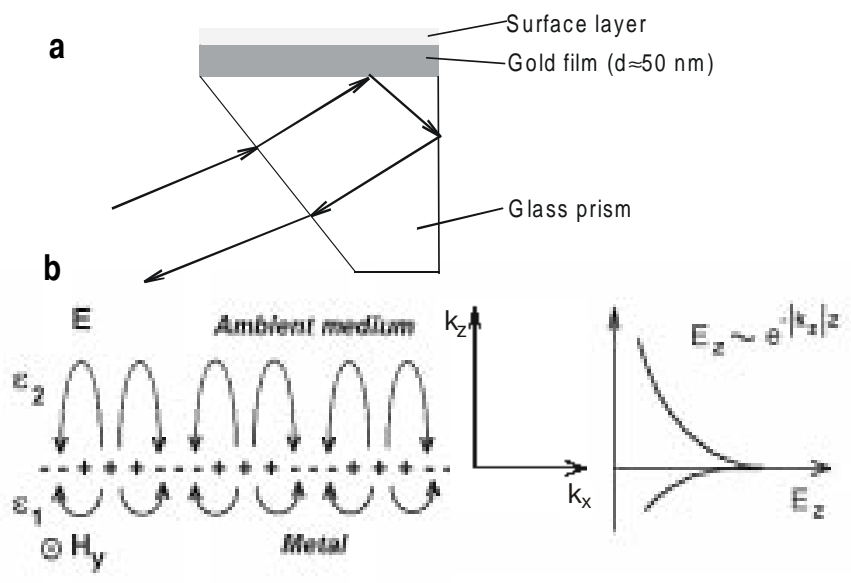

Fig. 2. Geometry of SPR measurements and model of surface layered structure (a) and distribution of electromagnetic field of the surface plasmon at the interface (b).
Fig. 1. Reference spectral dependencies of optical constants for gold thin films (A [13], B [14]) and bulk material (C [15]). 


\section{O.V. Rengevych et al.: Separate determination of thickness and...}

Under the above assumptions the total reflectivity of the model system can be calculated on the basis of Fresnel theory. For multilayer assembly, the most convenient computational scheme can be developed using Abeles matrix formalism [16]. The advantage of Abeles method is that each layer is fully represented by the single $2 \times 2$ matrix that does not depend on any other parts of the model under consideration. According to this method, the reflectance coefficient for $p$-polarized light can be given as follows:

$$
\mathrm{R}_{p}=-\left(u_{0 p}-Y_{p}\right) /\left(u_{0 p}+Y_{p}\right) \text {, }
$$

where $Y_{p}$ is an extended admittance (a high frequency surface conductivity) of a multi-phase system for the $p$-polarized wave. This quantity is defined as a ratio of tangential components of electric and magnetic vectors respectively at the interface that may be derived from the matrix expression:

$$
\left(\begin{array}{c}
1 \\
Y_{p}
\end{array}\right)=\left(\prod_{j=1}^{J-1}\left\|\begin{array}{cc}
\cos \delta_{j} & \left(i / u_{j p}\right) \sin \delta_{j} \\
i u_{j p} \sin \delta_{j} & \cos \delta_{j}
\end{array}\right\|\right) \cdot\left(\begin{array}{c}
1 \\
u_{J p}
\end{array}\right)
$$

Here $J$ is a total number of layers, including the ambience, $\delta_{j}$ is a phase thickness:

$$
\delta_{j}=2 \pi N_{j} \frac{d}{\lambda} \cos \phi_{j}
$$

and $u_{j p}$ is an admittance for $p$-polarized wave of the $j$-th layer:

$$
u_{j p}=N_{j} / \cos \phi_{j}=N_{j}^{2} / \sqrt{N_{j}^{2}-N_{0}^{2} \sin ^{2} \phi_{0}} .
$$

Here $N_{j}=n_{j}-i k_{j}$ is a complex refractive index, $\phi_{j}$ is an angle of incidence inside the $j$-th layer, $\lambda$ is a wavelength in vacuum, $d_{j}$ is a layer thickness, and $\phi_{0}$ is the external angle of incidence. Admittances $u_{p 0}$ and $u_{p J}$ are related to the ATR prism and to the ambience respectively.

Considering the experimental points of the resonance curve (or its characteristic parameters $\theta_{R}, \Delta \theta_{1 / 2}$, and $R_{\min }$ ) as a set of numerical data and equating them to the theoretical values given for the same angles of incidence by the expressions (1-4), one can calculate the set of model parameters $(n, k, d)$ that fits the experimental data. It is evident that the set of nonlinear equations obtained requires numerical procedure to find solution. Computing schemes usually employed for this purpose are known as optimization methods.

Generally, the optimization scheme consists in minimization of suitable objective function and typically includes the following steps. First, a set of theoretical datapoints is calculated for a tentative starting set of model parameters $\mathbf{x}$ ( $\mathbf{x}$ being $n, k$ and $d$ for different layers). Then the objective function to be minimized, $\mathbf{F}(\mathbf{x})$, is calculated. This function usually is taken in the form:

$\mathbf{F}(\mathbf{x})=\sum_{i}\left[\mathrm{R}_{i}(\mathbf{x})-R_{i}^{0}\right]^{2}$, where $\mathrm{R}_{i}(\mathbf{x})$ is the computed reflectivity, $R_{i}^{0}$ is the experimental one, and the subscript $i$ denotes the datapoint number. On the next step, the increments to the model parameters decreasing the objective function are found. This procedure is applied repeatedly until the local minimum of $\mathbf{F}(\mathbf{x})$ is reached.

Efficiency of these calculations depends on the minimization algorithm employed. In view of the fact that unambiguous solution can be found for only few model parameters by physical limitations, Nelder-Mead optimization algorithm [17] was chosen as the most effective for the low-dimensional problems and capable of minimizing the valley-shaped fitting functions.

The approach outlined above was accomplished using a specially designed software, that allows for a theoretical model to be easily modified in accordance with the conjectural structure of the system under study. The subset of model parameters to be processed by the optimization procedure could be assigned in arbitrary way for extracting the desired unknown data. Additionally, provision was made for the two-dimensional crossections of the fitting function to be mapped for any pair of model parameters. Target function maps were used for pictorial evaluation of possible ambiguity and precision of the solution found with the optimization procedure.

\section{Experimental}

The measurements were performed with the Plasmon-04 SPR spectrometer of proprietary design (Fig.3). This instrument utilizes attenuated total reflection (ATR) prism coupling in the Kretschmann - Raether geometry [9] for excitation of surface plasmons. The prism is mounted on the swivel carriage that rotates about the horizontal axis lying in the plane of the working facet of the prism. Rotation is performed automatically by the computer-controlled precision rotating mechanism. Maximum angle scan range is \pm 17 angular degrees. A solid-state laser $(\lambda=670 \mathrm{~nm})$ serves as a modulated light source. The reflected light intensity is measured using a Si photodiode operating in a zero-voltage

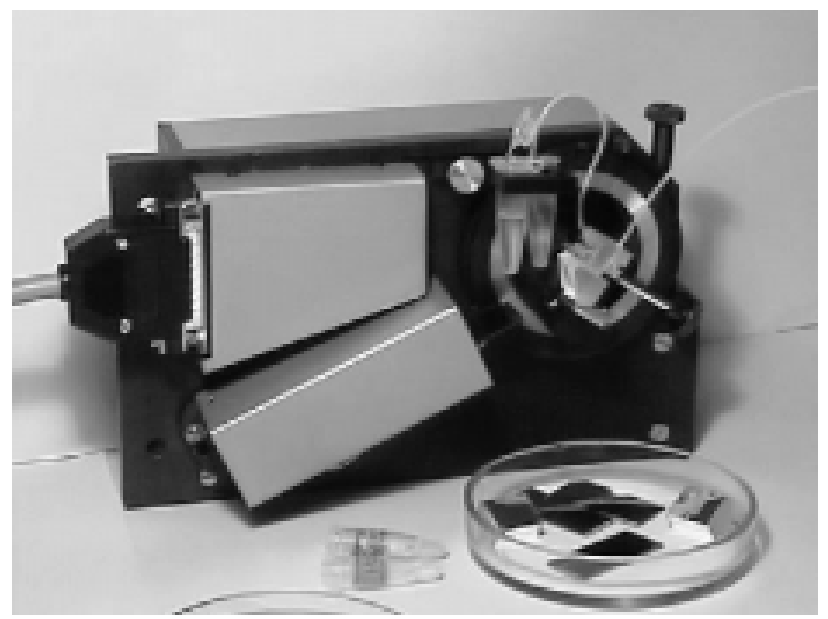

Fig. 3. SPR spectrometer Plasmon 04. 


\section{O.V. Rengevych et al.: Separate determination of thickness and...}

mode. As it has been shown previously [18], correct correlation of experimental SPR data with the results of numerical modeling requires the angle of incidence to be accurately measured. To satisfy this requirements, the Plasmon-04 instrument features a facility for measuring the absolute value of the angle of incidence onto the working facet of the prism utilizing an original self-adjustment autocollimation principle described in detail elsewhere [18].

In this study prisms carrying gold thin film directly deposited on their working facet were used. Gold films were prepared by vacuum deposition of 99.999 pure Au upon the surface of the prism. Before Au deposition, glass surfaces were cleaned subsequently in $\mathrm{NH}_{4} \mathrm{OH}: \mathrm{H}_{2} \mathrm{O}_{2}: \mathrm{H}_{2} \mathrm{O}$ and $\mathrm{HCl}: \mathrm{H}_{2} \mathrm{O}_{2}: \mathrm{H}_{2} \mathrm{O}$ solutions, both of $1: 2: 2$ by volume concentration, for $5 \mathrm{~min}$. at a boiling temperature, thoroughly rinsed in a bidistilled water, and dried in a flow of a pure nitrogen. Gold was thermally evaporated from a Mo heater and deposited at a rate of $1.0-1.5 \mathrm{~nm} / \mathrm{sec}$ immediately onto a glass surface kept at a room temperature. The total gold layer thickness was within $45-55 \mathrm{~nm}$. This technique resulted in a smooth hydrophobic surface with a limiting wetting angle of $80^{\circ}$. Since present study was focused on evaluation of film characterization reliability, measures has been taken to minimize uncontrollable variations of film parameters. Therefore to ensure the refractive index of gold films of different thickness to be identical, gold was deposited simultaneously on the prisms undergoing the same processing cycle, the only difference being the deposition time.

With these prisms, the procedure of determination of overlayer parameters from SPR measurements was attempted. Overlayers were formed by thermal vacuum evaporation of Calix [8]arene upon the gold films. Calixarenes are considered as promising materials for chemical sensing due to their interesting adsorption properties. SPR measurements on calixarene films were performed in air. Glass prisms $(n=1.5136)$ for studies with and without calixarene layers were prepared in pairs, the gold layer deposited on both prisms in the same pair being of equal thickness.

To enable determination of absolute values of reflectivity, the same ATR prisms without gold films were used for calibration. Reflectivity curve measured with the calibration prism was supposed to correspond to a $100 \%$ reflection and the SPR angular dependencies were then normalized with respect to this calibration curve.

To evaluate precision of determination of gold parameters, a set of measurements was carried out using surrounding media with different refractive index: water (1.337), isopropyl alcohol (1.382), and isobutyl alcohol (1.402). Glass prisms with $n=1.6160$ and refractive angle 65 degrees were used for these measurements. Isopropyl alcohol was high purification grade, isobutyl alcohol was "pro analysi" grade, water was twice distilled using a quartz distiller. Refractive indexes were measured using a standard refractometer IRF-22 at the same temperature as for the SPR measurements.

Independent measurement of gold film thickness and characterization of its surface morphology was performed by atomic force microscopy using NanoScope Dimension
3000 instrument ( Digital Instruments). For this purpose a groove was etched through the mask in the gold film using $\mathrm{I}: \mathrm{KI}$ aqueous solution.

\section{Results and discussion}

First we consider how does the uniquity and precision of characterization of the metal film depend on the angle range the SPR resonant curve is measured over. It will be shown that improper choice of this angle range may result in ambiguity in determination of the real part of the refractive index $n$ and the film thickness $d$. Calculated resonance curves for metal layer with different thickness and complex refractive index are shown in Fig. 4a-c. Wavelength $\lambda=632.8 \mathrm{~nm}$ and refractive indices of the ambient medium and the prism of 1.333 and 1.616 respectively were assumed for calculations. Comparison of these curves pictorially demonstrates what the ambiguity in $n$ and $d$ is due to when extracting separately thickness and optical constants of surface layer from the SPR experiment. Namely, increase in $n$ results in a similar change of the resonant curve as decrease in $d$ does, with the only exception of the portion of the resonant curve near the total internal reflection angle. The step between the plateaus in the curve below and up the total reflection angle increases with decrease in $d$ but does not depend on $n$. This difference become more pronounced if absolute values of reflectivity instead of normalized resonant curves are compared. In other words, dependency of the resonant curve on $n$ and $d$ parameters is near-degenerate, the degeneracy being less if angles below the total reflection are taken into consideration and absolute values of reflectivity are measured.

In line with this conclusion, the $n-d$ target function map for the resonant curve that includes the angle interval below the total reflection angle shows two minimums of considerably different depth (Fig.5a) while the minimums in the map for the curve with this interval being excluded are nearly equal (Fig. 5b). It should be noted that the above minimums correspond fairly well to two different sets of $\mathrm{Au}$ refractive indices shown in Fig.1. From the curve (a) the optical constants for $\lambda=670 \mathrm{~nm}$ may be estimated at $N^{*}=0.21+i 3.75$, and from the curve (c) - at $N^{*}=0.12+i 3.45$.

These conclusions are illustrated by Fig. 6 where two SPR curves are shown, the first curve being calculated for the model layer with $n_{A u}=0.2, k_{A u}=3.7, d_{A u}=55 \mathrm{~nm}$ and the second curve being obtained as the best fit to the first one when using the set of layer parameters $n_{A u}=0.1$, $k_{A u}=3.5, d_{A u}=50 \mathrm{~nm}$ as an initial approximation for the optimization procedure. This set corresponds to the point in the space of layer parameters located nearby the false minimum of the target function (see Fig.5), so that it is just this minimum the optimization process will converge to. The best fit was reached for $n_{A u}=0.11, k_{A u}=3.7, d_{A u}=42.5$, the correspondent resonant curve being only slightly different from the target one within the angle range from 60 to 64 degrees. In fact, this difference is comparable with the typical experimental errors. The difference, nevertheless, becomes more significant when a wider angle range is consi- 


\section{O.V. Rengevych et al.: Separate determination of thickness and...}
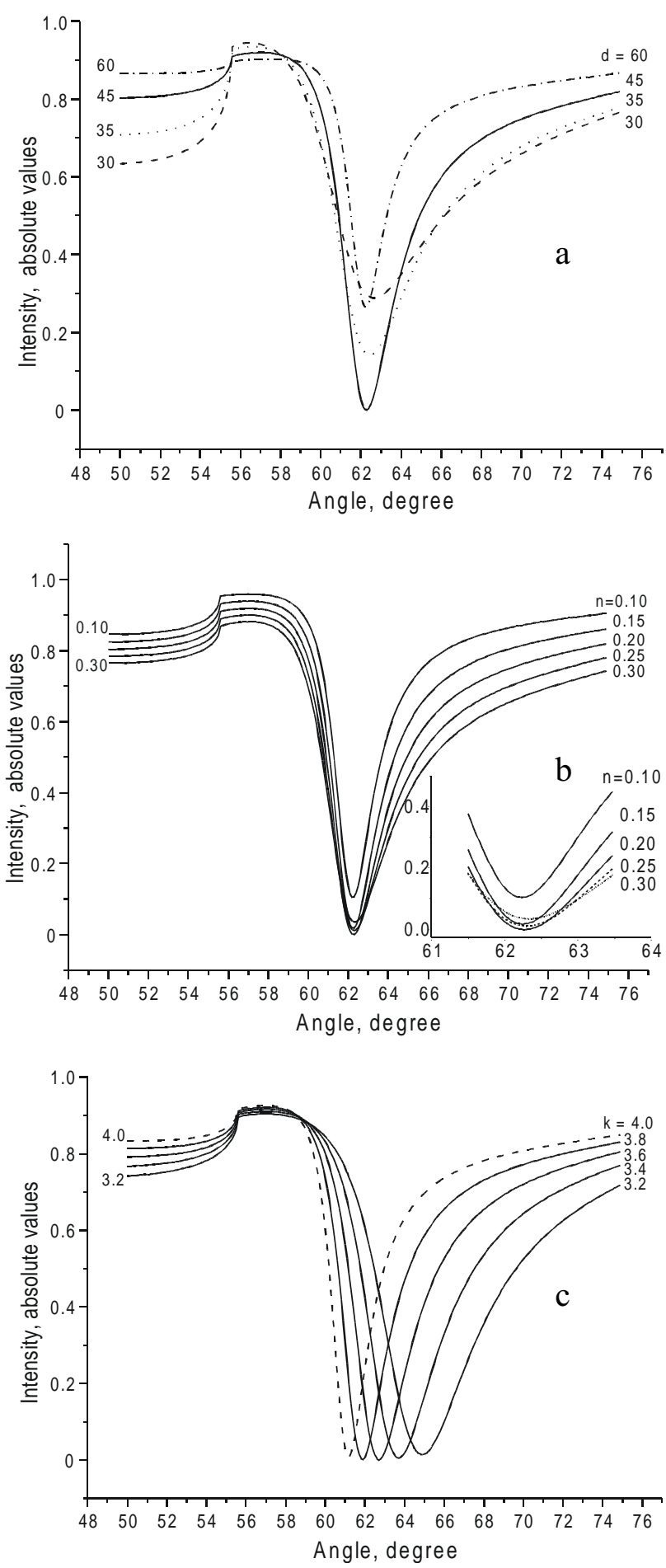

Fig. 4. Calculated SPR curves for metal layer with different thickness (a), real part of refractive index (b), imaginary part of refractive index (c) .

dered, especially in the region close to the total internal reflection angle. Therefore, unambiguous characterization of the metal film requires the measurements to be taken over an angle range that includes this region.

Nevertheless, from the viewpoint of sensor applications, the major consequence of the SPR phenomenon is related to its capability of probing and real time monitoring of a
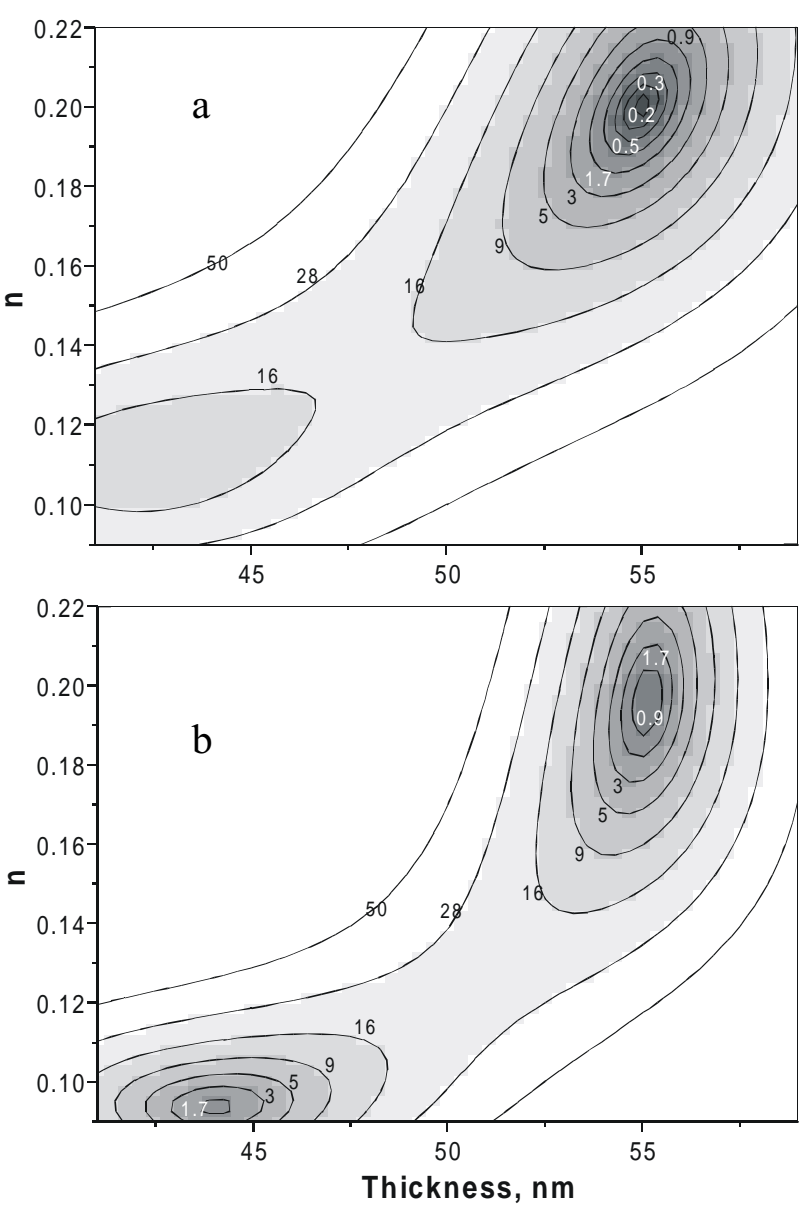

Fig. 5. $n$ - $d$ target function map for the resonant curve that includes (a) and excludes (b) the angle interval below the total reflection angle.

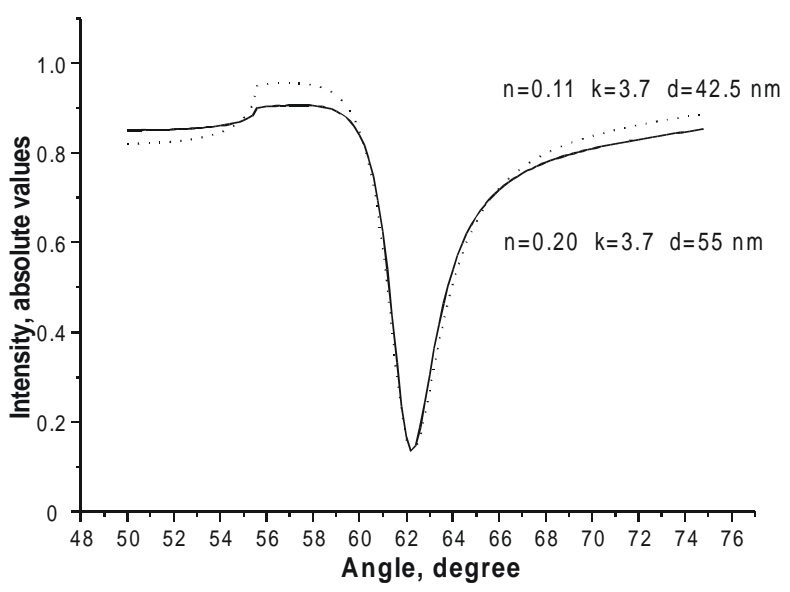

Fig. 6. SPR curves for two minimums of target functions map, shown in Fig.5a.

sensitive overlayer deposited above the SP-supporting metal film. Correct determination of the overlayer parameters requires the gold film optical constants and thickness to be measured precisely. To evaluate its precision, the SPR measurements were taken on the same gold layers with different refractive indices of the surrounding media (Fig.7). Gold film parameters extracted using the above described 


\section{O.V. Rengevych et al.: Separate determination of thickness and...}

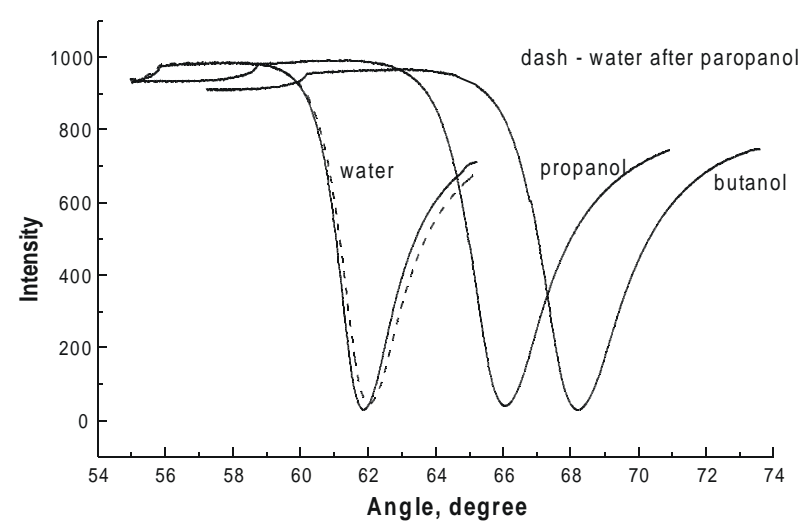

Fig. 7. Experimental SPR curves for the surrounding media with different refractive indices.

procedure are summarized in Table 1 . It can be seen from the Fig.7 that some quantity of irreversibly adsorbed isopropyl alcohol remains on the gold surface. An independent measurement of gold layer thickness performed by atomic force microscopy, $d_{A u}=51.1 \mathrm{~nm}$ (Fig. 8), falls within values determined from SPR measurements. Nevertheless, gold film parameters determined in different media are not equal. This discrepancy may be attributed to inadequate theoretical model used for modeling of optical properties of the layered structure. It has been demonstrated long ago that plasmon scattering on surface roughness influences the plasmon resonance curve. This effect was first investigated using $\mathrm{CaF}_{2}$ sublayer to enhance surface roughness [19].

Table 1. Calculated values of gold film parameters for different environments.

\begin{tabular}{|c|c|c|c|c|c|}
\hline \multicolumn{2}{|c|}{ Environment } & \multicolumn{4}{|c|}{ Gold film parameters } \\
\hline \multicolumn{6}{|c|}{ True minimum values } \\
\hline & $n_{\text {env }}$ & $n_{A u}$ & $k_{A u}$ & $d_{A u}, \mathrm{~nm}$ & $z$ \\
\hline Butanol & 1.402 & 0.179 & 3.92 & 51.2 & 0.694 \\
\hline Propanol & 1.382 & 0.197 & 3.91 & 50.9 & 1.04 \\
\hline Water & 1.337 & 0.173 & 3.87 & 52.2 & 1.51 \\
\hline $\begin{array}{c}\text { Water (after } \\
\text { propanol) }\end{array}$ & 1.337 & 0.188 & 3.83 & 52.8 & 1.31 \\
\hline \multicolumn{6}{|c|}{ False minimum values } \\
\hline & $n_{e n v}$ & $n_{A u}$ & $k_{A u}$ & $d_{A u}, \mathrm{~nm}$ & $z$ \\
\hline Butanol & 1.402 & 0.132 & 3.91 & 47.4 & 1.62 \\
\hline Propanol & 1.382 & 0.137 & 3.91 & 46.5 & 2.97 \\
\hline Water & 1.337 & 0.128 & 3.87 & 48.1 & 2.09 \\
\hline $\begin{array}{r}\text { Water (after } \\
\text { propanol) }\end{array}$ & 1.337 & 0.126 & 3.83 & 47.2 & 2.18 \\
\hline
\end{tabular}

The similar approach was then exploited by many other researchers. In [20], an attenuated total reflection and rearside light emission was measured on $50 \mathrm{~nm}$ thick gold films evaporated on glass substrates previously coated with $\mathrm{LiF}$ layers of 0-500 nm thickness to enhance surface roughness. The complex dielectric constant of metal films was determined as a function of the fluoride thickness. Both the average grain diameter $(\delta)$ and the correlation length $(\sigma)$ were calculated from the angular distribution of the emission intensity and, independently, from atomic force microscope images. Roughness amplitudes $\delta$ were found to be proportional to the fluoride thickness up to $350 \mathrm{~nm}$. It was demonstrated that the effective dielectric functions and surface plasmon wavevectors of gold layer change drastically under the influence of increasing roughness amplitudes.

A theoretical description of the attenuation of surface polaritons by roughness on the surface was presented in [21]. In the presence of surface roughness, the surface polariton is attenuated by two processes. It may lose energy by radiation into the vacuum, or by scattering into other surface-polariton states. Through application of a formalism developed to describe roughness-induced scattering and absorption of a plane electromagnetic wave incident on a surface, contribution to the attenuation rate from the two processes was calculated. These studies led to a conclusion that light emission induced by surface roughness is responsible mainly for increase in the resonant curve angular width, while the scattering of surface polaritons into other polariton states contributes also to dispersion of the surface polaritons and is responsible for the resonant curve shift.

Another possible cause of discrepancy may originate from insufficiency of Freshnel approach pointed out in [2225]. The usual Fresnel theory of reflection and transmission was shown to be incorrect when applied to materials in which longitudinal (electrostatic) polarization waves, such as the bulk plasma wave (plasmon), may propagate, and new macro - and microscopic theories were proposed.

Finally, determination of calixarene film parameters from the SPR measurements was attempted. Parameters of gold layer were determined previously using measurements on the same gold film before deposition of calixarenes. The results summarized in Table 2 are of reasonable value but essentially different for different gold layer thickness.

Table 2. Gold layer parameters for true and false minimums.

\begin{tabular}{cccccc}
\hline \hline & $\begin{array}{c}\text { Prism } \\
\text { number }\end{array}$ & $\begin{array}{c}\text { minimum } \\
\text { number } \\
\text { (true/false) }\end{array}$ & $n_{A u}$ & $k_{A u}$ & $\begin{array}{c}d_{A u}, \\
\mathrm{~nm}\end{array}$ \\
\hline 1 & 1 & 1 (true) & 0.209 & 3.82 & 52.6 \\
2 & 1 & 2 (false) & 0.1175 & 3.87 & 45.0 \\
3 & 2 & 1 (true) & 0.209 & 4.07 & 32.7 \\
4 & 2 & 2 (false) & 0.375 & 4.00 & 43.1 \\
\hline \hline
\end{tabular}

This is not surprising in view of the above consideration. Therefore a question arises what kind of information about overlayers can be extracted with higher precision from the SPR measurements. It should be noted that in many instances such as immunological or DNA hybridization studies the refractive index and the thickness of overlayer as themselves are of little consequence, more important figure being the total quantity of adsorbed species. A standard protocol for experiments of this type with the use of the SPR spectroscopy includes immobilization of one of the interacting counterparts on a loose easily permeable matrix such as 


\section{O.V. Rengevych et al.: Separate determination of thickness and...}
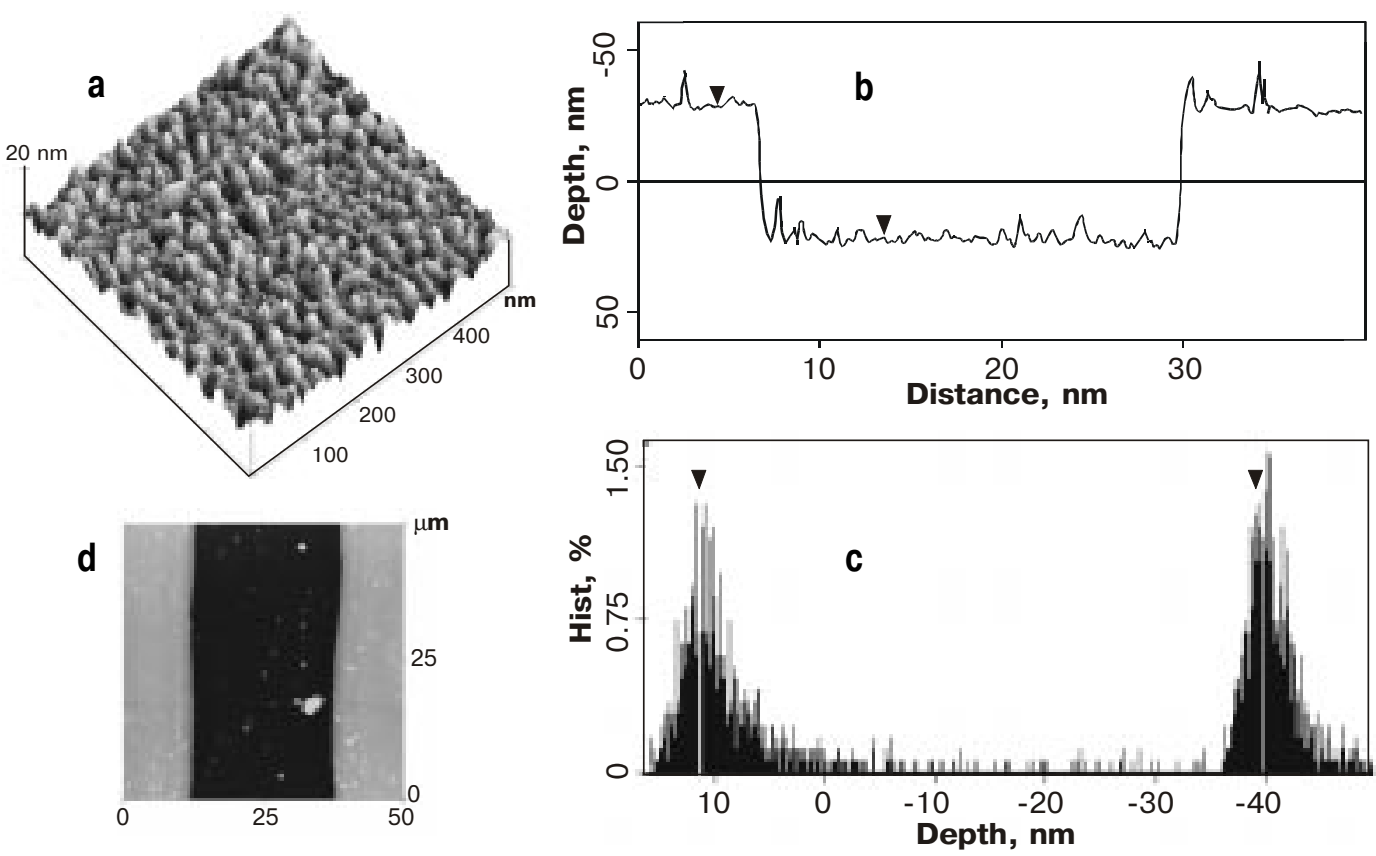

Fig. 8. Results of AFM studying gold layer deposited on the ATR prism. Surface morphology of the layer (a), section analysis (b), depth analysis (c), and top view (d) of a pattern where the gold film was stripped. Vertical distance between markers corresponds to 51.1 nm.

carboxymethylated dextrane deposited over a gold film $[26,27]$. Another counterpart is dissolved in a suitable solvent. In the course of the reaction the dissolved species are being captured by immobilized ones thus resulting in increase of a total amount of adsorbed molecules within the matrix volume. The concentration of the captured species, $\Gamma$, is then estimated using the correspondent refractive index increment, $d n / d c$ [28]:

$\Gamma=d\left(n-n_{0}\right) /(d n / d c)$.

For instance, a typical value of $d n / d c$ for immunoglobulins is $0.188 \mathrm{~cm}^{3} / \mathrm{g}$. Under this analysis, $n-n_{0}$ is assumed to be proportional to the angular shift of the resonant curve. The above approach obviously involves a degree of uncertainty. One might conclude that, when using the $d n / d c$ value it is necessary that no other parameter than the refractive index $n$ changes as a result of the reaction, an assumption that scarcely may be valid. It will be shown here that it is not the case since the $n, d$ parameters of layers with different densities but containing equal quantities of the same compound are interdependent. This dependency the most suitably may be treated in terms of molecular refraction. Hence the total amount of the adsorbed molecules can be evaluated correctly if $n, d$ parameters are known, but, as it was demonstrated earlier, separate extraction of these parameters suffers from a relatively poor precision. Nevertheless, the analysis presented below shows that errors in pairs of $n, d$ values extracted from the SPR measurements are mutually dependent in such a way that these pairs may be used instead of correct parameters for quantitative determination of adsorption.

Denote the molecular refraction by $A$, and the molecular refraction per unit volume by $A_{V}, A_{V}=A\left(N / N_{A}\right)$, where $N$ is a number of molecules per unit volume, and $N_{A}$ the Avogadro number. When compounds with different $A_{V}$ are mixed together, the resulting $A_{V}$ value is given as follows:

$A_{V}=\left(A_{V 1} \cdot V_{1}+A_{V 2} \cdot V_{2}\right) /\left(V_{1}+V_{2}\right)$.

The $A_{V}$ value is related to the refractive index by the following expression:

$A_{V}=\left(n^{2}-1\right) /\left(n^{2}+2\right)$.

From (8), $A_{V}$ can be calculated for both the solvent and the substance to be analyzed provided that their refractive indices are known. Assuming then the overlayer to be a mixture of the substance and the solvent, a resulting $A_{V}$ may be found from (7) and the correspondent refractive index from (8). Dependence of the refraction $A_{V}$ on $d$ calculated from (7) for the model substance and the solvent refractive indices of 1.7 and 1.333 respectively is presented in Fig.9. Initial thickness of $20 \mathrm{~nm}$ was assumed for the overlayer containing pure model substance only. For this layer the SPR resonant

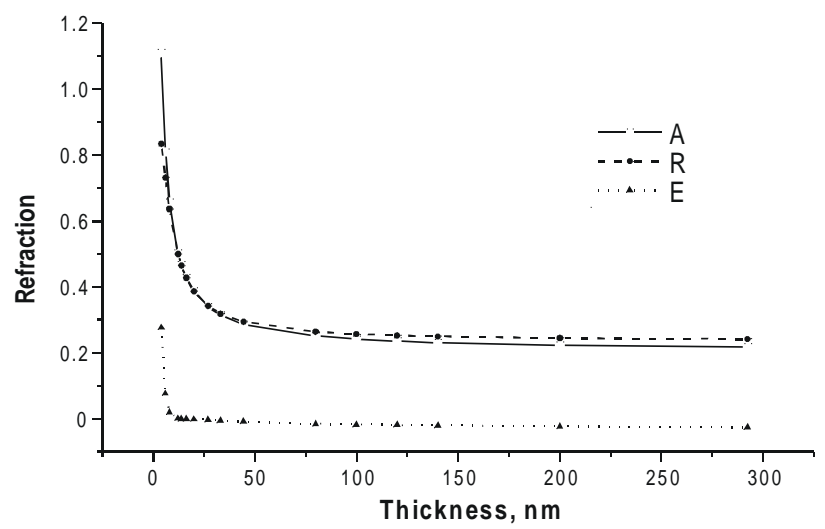

Fig. 9.Molecular refraction versus dielectric film thickens. A - calculated refraction of model dielectric film of mixed composition; R - refraction, calculated using $n$ and $d$ pairs extracted from the model SPR curve; E difference between dependencies $\mathrm{A}$ and $\mathrm{R}$. 


\section{O.V. Rengevych et al.: Separate determination of thickness and...}

curve was also calculated. Then, assuming different values for the layer thickness, correspondent values of $n$ were extracted from this resonant curve using fitting procedure. Molecular refraction values calculated for these $n$ are also plotted on Fig.9. One can see that from about 15 to $50 \mathrm{~nm}$ both dependencies practically coincide, the difference becoming more pronounced for thicknesses exceeding 70 $80 \mathrm{~nm}$. Hence, one can conclude that even when separate extraction of $n, d$ values from SPR data suffers from poor precision, correlation between these values nevertheless enables determination of the total amount of the adsorbed material, provided that the apparent layer thickness in significantly less then the penetration depth of the evanescent electromagnetic field of surface plasmons into the surrounding medium.

\section{Conclusions}

Reliability and precision of characterization of surface layers by SPR method has been analyzed with the focus at the separate extraction of optical constants and thickness of surface coatings and determination of the total quantity of deposited material. Numerical processing of experimental SPR curves based on fitting procedure was employed. Computational scheme for modeling the SPR resonance dependence for multilayer assembly, based on Abeles matrix formalism, is presented. An optimal angle range for SPR measurements on metal films was found. It was demonstrated that improper choice of this range may result in ambiguity in determination of the real part of the refractive index $n$ and the film thickness $d$.

Nevertheless, it is shown that the total quantity of material in the film can be estimated with reasonable accuracy even when correct separate extraction of $n$ and $d$ parameters is hampered by experimental errors and inadequacy of theoretical model of layered system. This is due to the fact that errors in pairs of $n, d$ values extracted from the SPR measurements are mutually dependent in such a way that these pairs may be used instead of correct parameters for quantitative determination of adsorption provided that the layer thickness is much smaller than the wave length.

\section{Acknowledgements}

Authors are grateful to S. Zynio for vacuum deposition of gold films and to Dr. G.Beketov for fruitful discussions.

\section{References}

1. C.Nylander, B.Liedberg, T.Lind, Gas detection by means of surface plasmon resonance // Sensors and Actuators 3, pp.79-88 (1982/83).

2. B.Liedberg, C.Nylander, I.Lundstrom, Surface plasmon resonance for gas detection and biosensing // Sensors and Actuators 4, pp.299-304 (1983).

3. M.T.Flanagan, R.H.Pantell, Surface plasmon resonance and immunosensors // Electr.Lett. 20, pp.968-972 (1984).

4. K.A.Peterlinz, R.Georgiadis, In situ kinetics of self-assembly by surface plasmon resonance spectroscopy // Langmuir. 12, pp.4731-4740 (1996).
5. E.F.Aust, S.Ito, M.Savodny and W.Knoll, Investigation of polymer thin films using surface plasmon modes and optical waveguide modes // Trends in Polymer Science 2(9), pp.313-323 (1994).

6. B.S.F.Altenburg, H.E.deBruijn, R.P.H.Kooyman, and J.Greve, Determination of thickness and dielectric constant of the transparent dielectric layers // Optical communications 82, pp.425-432 (1991).

7. R.M.A.Azzam, N.M.Bashara, Ellipsometry and polarized light. North-Holland Publishing company, Amsterdam (1977).

8. H.Raether, Surface plasmons on smooth and rough surfaces and on gratings. Springer Tracts in Modern Physics, Springer-Verlag, Berlin- Heidelberg (1988).

9. K.Welford, The method of attenuated total reflection, in: Surface plasmon polaritons, IOP Short Meetings series, N9, IOP Publishing Ltd. pp.25-78 (1988).

10. F.Forstmann and H.Stenschke, Electrodynamics at metal boundaries with inclusion of plasma waves // Phys. Rev. Lett. 38(23), pp.13651368 (1977).

11. Y.Borensztein, Investigation of nonlocal electromagnetic phenomena in thin silver films near the plasma frequency // J.Opt.Soc.Am. 73(1) pp.80-86 (1983).

12. R.A.Innes, J.R.Sambles, Optical characterization of gold using surface plasmon-polaritons // J.Phys.F: Met. Phys. 17, pp.277-287 (1987).

13. Marie-Luce Theye. Investigation of the optical properties of Au by means of thin semitransparent films // Phys. Rev. B. 2(8) pp.30603078 (1970).

14. Wilford N. Hansen, Electric fields produced by the propagation of plane coherent electromagnetic radiation in a stratified medium // J.Opt. Soc. Am. 58(3) pp.380-390 (1968).

15. W.H. Piller, Lead-Tin-Telluride, Handbook of Optical Constants of Solids, Academic Press, New York, E.D. Palik, Ed. 2. (1991).

16. F.Abeles, Recherches sur la propagation des ondes electromagnetiques sinusoedales dans les milieux stratifies. - Application aux couches mines // Ann. Physique 5, pp.596-706 (1950).

17. J.A.Nelder, R.Mead, A simplex method for function minimization // The Comp. Journal 7, pp.308-313 (1965).

18. G.V.Beketov, Yu.M.Shirshov, O.V.Shynkarenko, V.I.Chegel, Surface plasmon resonance spectroscopy: prospects of superstrate refractive index variation for separate extraction of molecular layer parameters // Sensors and Actuators B. 48, pp.432-438 (1998).

19. E.Burstein, W.P.Chen, Y.J.Chen, A.Harstein. Surface polaritons - propagating electromagnetic modes at interfaces// J. Vac. Sci. Technol. 11(6) pp.1004-1019 (1974).

20. A.Hoffman, Z.Lenkefi, Z.Scentirmay, Effect of roughness on surface plasmon scattering in gold films // J.Phys.:Condens.Matter. 10, pp.5503-5513 (1998)

21. D.L.Mills, Attenuation of surface polaritons by surface roughness // Phys. Rev.B 12(10), pp.4036 - 4046 (1975).

22. A.R.Melnyk, M.J.Harrison, Theory of optical excitatiom of plasmons in metals // Phys.Rev.B. 2(4), pp.835-850 (1970).

23. A.R.Melnyk, M.J.Harrison, Optical excitatiom of plasmons in metals:microscopic theory // Phys.Rev.B. 2(4), pp 851-857 (1970).

24. Y.Borensztein, investigation of nonlocal electromagnetic phenimena in thin silver films near plasma frequency // J.Opt.Soc.Am. 73(1), pp.80-86 (1983).

25. F.Frostmann, H.Stenschke, Electrodynamics at metal boundaries with inclusion of plasma waves // Phys.Rev.Lett. 38(32), pp.1365-1368 (1977).

26. S.Lofas, B.Johnsson, A novel hydrohel matrix on gold surfaces in surface plasmon resonance sensors for fast and efficient covalent immobilization of ligands // J.Chem.Soc.Chem.Commun. 21, pp.1526528 (1990).

27. M.C.Millot, F.Martin, D.Bousquet, B.Sebille, Y.Levy. A reactive macromolecular matrix for protein immobilization on a gold surface. Application in surface plasmon resonance // Sensors and Actuators B. 29, pp.268-273 (1995).

28. M.Malmstein, Ellipsometry studies of protein layers adsorbed at hydrophilic surfaces // J.Colloid and Interface Science 166, pp.333342 (1994). 